\section{Revista \\ Brasileira \\ de Enfermagem \\ REBEn}

HISTÓRIA DA ENFERMAGEM

\title{
Memória da implantação da Graduação em Enfermagem na Unicamp
}

\author{
Creation memory of the Nursing Undergraduate Course at the University of Campinas
}

Memoria de la implantación del Curso de Graduación en Enfermería en la

Universidad de Campinas

Débora Martins Zulske

Acadêmica do Curso de Enfermagem da Faculdade de Ciências Médicas, Unicamp,

Campinas, SP.

\section{Márcia Regina Nozawa}

Doutor em Saúde Pública. Professor doutor do Departamento de Enfermagem da Faculdade de

Ciências Médicas, Unicamp, Campinas, SP.

\section{RESUMO}

Objetivou-se reconstituir a história de implantação da Graduação em Enfermagem na Faculdade de Ciências Médicas, Universidade Estadual de Campinas, ocorrida em 1978, através de história oral, pela insuficiência de fontes documentais pertinentes. Enfermeiros que participaram direta e ativamente nesse processo foram os sujeitos de pesquisa. A faculdade de enfermagem, legalmente prevista desde 1966, nunca foi efetivada. Para responder à mínima organização necessária, instituiu-se apenas um Departamento de Enfermagem, quatro anos após o início do curso. O interesse e a iniciativa de instalação do curso partiu de um enfermeiro titulado, externo à universidade, contratado especificamente para esse fim. Este fato explica a marca pessoal, pouco institucional, que caracterizou, de forma predominante, o momento de instalação e a continuidade desse curso. Descritores: Enfermagem; Programas de graduação em enfermagem; História

\section{ABSTRACT}

The objective of this study was to reconstitute the history of the creation of the Nursing Undergraduate Course at the Medical Sciences School, University of Campinas, occurred in 1978, through an unofficial history, due to the insufficiency of pertinent documental sources. Nurses who participated directly and actively in this process were the subjects of the research. The School of Nursing, legally planned since 1966, was never established. To answer to the minimum necessary organization, it was instituted only one Department of Nursing, four years after the beginning of the course. The interest and the initiative to establish the course started from a titled nurse, outside of the university, hired specifically for this purpose. This fact explains the personal trace, not very institutional, that characterized in a predominant way the moment of creation and continuity of this course. Descriptors: Nursing; Education, nursing, diploma programs; History.

\section{RESUMEN}

El objetivo de este artículo es reconstituir la implantación de la graduación en enferemería de la Facultad de Ciencias Médicas, de la Universidad Estadual de Campinas, que ocurrió en 1978, por medio de la historia hablada, por no haber fuentes documentales pertinentes. Los enfermeros que participaron directa y activamente en ese proceso fueron los sujetos de la pesquisa. La Facultad de Enfermería, legalmente prevista desde 1966, nunca fue efectivada. Para responder a la mínima organización necesaria, solamente se instituyó un Departamento de Enfermería, cuatro años después del inicio del curso. El interés y la iniciativa de la instalación del curso ocurrió por un enfermero graduado, externo a la faculdad, contratado especialmente para este trabajo. Este facto explica la marca personal y no institucional, que caracterizó de manera predominante el momento de la instalación y de la continuidad de este curso.

Descriptores: Enfermería; Programas de graduación en enfermería; Historia.

Zulske DM, Nozawa MR. Memória da implantação da Graduação em Enfermagem na Unicamp. Rev Bras Enferm 2006 jul-ago; 59(4):573-7.

\section{INTRODUÇÃO}

Com a Resolução $n^{0} 44$ de 1966, o Conselho Estadual de Educação do Estado de São Paulo(1) autoriza a instalação de uma faculdade de enfermagem na Universidade Estadual de Campinas (Unicamp) em 1966, ano em que se instala a pedra fundamental do campus da Universidade. Contudo, a Graduação em Enfermagem, bacharelado e licenciatura, teve início somente no ano de 1978, e conforma-se como tal dentro da Faculdade de Ciências Médicas (FCM), unidade de ensino também responsável pela graduação médica, a mais antiga da universidade, legalmente formalizada pela Lei Estadual $n^{0} 2.154$ de 30 de junho de 1953, mas autorizada a funcionar somente em $1963^{(2)}$. Mais recentemente, em 2002, teve início o curso de graduação em fonoaudiologia, numa parceria entre e
Submissão: 04/01/2006

Aprovação: 23/05/2006 
FCM e o Instituto de Estudos da Linguagem (IEL) da Unicamp e no ano de 2004, o curso de farmácia, com coordenação compartilhada entre a Faculdade de Ciências Médicas e os Institutos de Biologia e de Química.

A Unicamp, como entidade autárquica, foi legalmente instituída em dezembro de 1962, mas, efetivamente, passou a existir somente em 1966 quando incorporou a então Faculdade de Medicina de Campinas. Esta funcionou na Maternidade de Campinas e transferiu-se, posteriormente, para as instalações da Santa Casa de Misericórdia de Campinas, ali permanecendo até a construção do Hospital das Clínicas (HC). A obra do prédio do HC, iniciada em 1975, finalizou-se em 1986, muito embora 0 atendimento ambulatorial já funcionasse nas novas instalações desde $1979^{(2)}$.

De 1966 até 1978, Unicamp foi dirigida pelo professor Zeferino Vaz, nomeado reitor por decreto do governador do Estado, grande idealizador que aliava em seu perfil características autoritárias e paternalistas, imprimiu um estilo de administração pouco institucional. Os professores eram contratados por mérito, não havia concurso, a carreira docente não estava definida e as decisões na universidade eram tomadas caso a caso(3).

Em 1978, quando se encerra o período previsto de implantação, a Unicamp contava com cerca de 1000 docentes e 14 unidades de ensino (2):(3) ocorrendo a criação do Curso de Graduação em Enfermagem na universidade. Sabe-se que, a convite do então diretor da FCM, um enfermeiro não pertencente aos quadros da Unicamp recebeu a incumbência de formalizar o projeto de criação do Curso de Enfermagem e iniciar sua implantação.

O currículo adotado, e em vigor até o ano de 1996, correspondia em termos gerais, ao currículo mínimo para os cursos de graduação em enfermagem, instituído pelo Parecer n ${ }^{0}$ 163/72 do Conselho Federal de Educação(4).

O reconhecimento dos Cursos de Bacharelado e Licenciatura em Enfermagem ocorreu mediante Parecer $n^{0} 2038 / 81$ do Conselho Estadual de Educação e Portaria MEC nº 322, publicada em Diário Oficial da União em $20 / 08 / 82^{(5)}$.

Obedecendo ao currículo previsto, as disciplinas do tronco básico do Curso de Graduação em Enfermagem, concentradas no primeiro ano, foram ministradas, predominantemente, pelo Instituto de Biologia e também por alguns departamentos da FCM. A partir de 1979, quando se iniciou 0 oferecimento de disciplinas específicas de enfermagem, pertencentes ao tronco profissionalizante, houve a participação de diversos enfermeiros, entre eles, alguns originários do quadro de pessoal do então Hospital das Clínicas, visto que não havia um corpo docente constituído para esse fim.

A instalação posterior do Departamento de Enfermagem implicou a incorporação gradual e não institucional de enfermeiros para atender às necessidades de cada nova disciplina de graduação que ia sendo oferecida. A partir desse conjunto de profissionais, a criação oficial do Departamento de Enfermagem na estrutura da FCM, com dez docentes, ocorreria somente no ano de $1981^{(6)}$. Desde então, o Departamento de Enfermagem mantém-se subordinado à FCM, como o único departamento de ensino, entre os 16 existentes, exclusivamente composto por docentes não médicos.

O argumento central para a criação do curso de Graduação em Enfermagem da Unicamp era a necessidade emergente de formação de mão-de-obra para o Hospital das Clínicas, em fase de construção, e para a rede básica de saúde do município de Campinas em processo de implantação(1).

Contudo, o currículo adotado, oferecia uma formação compartimentalizada, por meio de disciplinas que reproduziam as especialidades médicas com ações de saúde, predominante, de caráter curativo e adequava-se ao modelo econômico excludente e concentrador de rendas de então, ou seja, a medicalização da saúde e tecnificação do ato médico e conseqüente expansão dos interesses capitalistas na saúde.

O curso, sempre ofertado em período integral, era passível de integralização em oito semestres, na modalidade bacharel ( 245 créditos ou 3675 horas), e em nove semestres para abranger a licenciatura, implicando acréscimo de 36 créditos ou 540 horas. Até 0 ano de 1999, 0 curso disponibilizava 30 vagas anuais.

Em outubro de 1983, realizou-se, por iniciativa de alunos, o primeiro seminário de avaliação curricular. Nessa ocasião, o Departamento de Enfermagem contava com 15 docentes, cinco deles admitidos naquele mesmo ano, entre os quais apenas dois portavam titulação acadêmica, um mestre e um livre docente, e outro era mestrando. Assim, o grupo de docentes compunha-se majoritariamente de graduados em Enfermagem, entre eles alguns especialistas. A admissão de professor específico para a Enfermagem em Saúde Pública ocorreu somente nesse ano. Outros dois seminários de avaliação foram realizados nos anos de 1985 e 1991, organizados pela própria Comissão de Ensino de Graduação, os quais trataram de identificar problemas e propor medidas para aprimorar o ensino ministrado ${ }^{(7)}$.

Nos últimos anos da década de 80 e primeiros da década de 90 , docentes de algumas disciplinas que tradicionalmente desenvolviam 0 ensino prático em campos hospitalares, tais como, fundamentos de enfermagem, enfermagem ginecológica e obstétrica e enfermagem cirúrgica, tomaram a iniciativa de estender o ensino a unidades básicas de rede municipal de saúde de Campinas e outros equipamentos sociais, tendo em vista a necessidade de desenvolver nos alunos determinadas competências que dificilmente eram possíveis em unidades de internação ou ambulatoriais de um hospital especializado pela suas características e finalidade ${ }^{(6)}$.

A constatação dessa mudança não oficializada aliada à crítica da inadequação do currículo, efetivada, principalmente, por docentes da área de enfermagem de saúde pública e saúde mental, reforçou a necessidade de iniciar um processo de revisão e reforma curricular. De modo mais organizado, no ano de 1993, por ocasião do evento comemorativo dos 15 anos do Curso de Graduação, iniciou-se um movimento de avaliação curricular que se estendeu pelos três anos subseqüentes e que resultou na proposta de um novo currículo para a graduação, implantada em $1997^{(7)}$.

No plano das proposições, o novo currículo de Graduação em Enfermagem da Unicamp, comparado ao anterior, previa uma subversão da ordem instituída pela inversão de eixos norteadores do ensino, ou seja, o aprendizado do processo saúde-doença partiria do enfoque coletivo em direção ao individual, pelo reconhecimento das condições sociais, políticas, culturais e biológicas de sua produção.

Somente em 1999 iniciou-se o programa de pós-graduação em enfermagem nível mestrado na Unicamp. Desde então, as exigências quantitativas de produção científica do corpo docente, impostas pelas instâncias oficiais de avaliação dos programas de pós-graduação, somadas aos indicadores institucionais de avaliação docente aplicados nos últimos anos, ou seja, de valorização da produtividade científica, acabaram por conformar uma maior preocupação e atenção dos professores nessa direção com prejuízos evidentes na docência, principalmente de graduação(8,9).

Em 2000, houve ampliação de dez vagas de graduação, conformando um ingresso anual de 40 alunos.

Atualmente, o Departamento de Enfermagem é composto por 22 docentes em exercício efetivo, três professores associados, 19 doutores e 15 enfermeiros da carreira assistencial que assumem atividades de ensino de graduação. Entre esses, dois doutores, dois doutorandos e sete mestres e dois mestrandos. 0 quadro de pessoal, já deficitário, apresentou redução drástica em virtude da aposentadoria de sete docentes, ou cerca de 30\% do corpo docente, somente no ano de 2003.

A proposição deste estudo situou-se entre as diversas iniciativas institucionais de recuperação da memória do Curso de Graduação em Enfermagem da Unicamp que completou seus 25 anos de existência em 2003 , tendo já conferido graduação a aproximadamente 530 enfermeiros. 


\section{OBJETIVO}

Reconstituir a história da implantação do Curso de Graduação em Enfermagem da Unicamp.

\section{METODOLOGIA}

O relato oral tem sempre se constituído, ao longo dos séculos, como a maior fonte humana de conservação e divulgação do saber, ou a maior fonte de dados para as ciências em geral ${ }^{(10)}$. A narrativa oral, após transcrição, se transforma num documento semelhante a qualquer outro texto escrito que serve de base para o trabalho de investigação.

A história oral recobre relatos acerca de fatos não registrados por outro tipo de documentação ou que se deseja completar. Ela pode ser colhida por meio de entrevistas realizadas de formas variadas, registrando a experiência de um só ou em coletividade, procurando-se, neste caso, atingir uma convergência de relatos sobre um mesmo acontecimento ou determinado período de tempo ${ }^{(10,11)}$. Representa, assim, a construção de uma abordagem alternativa à historiografia tradicional, na medida em que toma por objeto a vida cotidiana, o homem comum, os fenômenos sociais não escritos, não formalizados ${ }^{(10,11)}$.

A coleta de dados dessa pesquisa foi feita por meio de entrevista orientada por um roteiro de questões, em consideração aos objetivos propostos, e através da consulta à documentos oficiais, referentes ao processo de Implantação do Curso de Graduação em Enfermagem ${ }^{(1)}$ e à constituição do Departamento de Enfermagem na Faculdade de Ciências Medicas da Unicamp ${ }^{(12)}$.

O projeto recebeu aprovação do Comitê de Ética em Pesquisa da FCM, Unicamp, em 19/08/2003, mediante o parecer de $n^{0} 291 / 2003$. No momento da entrevista, os depoentes tomaram conhecimento do projeto de pesquisa e do roteiro de questões orientadoras e também assinaram 0 Termo de Consentimento Livre e Esclarecido.

As fontes secundárias de pesquisa, ou seja, os documentos institucionais, contêm indicações que permitiram identificar seis enfermeiros que participaram do momento histórico de criação do Curso de Enfermagem ${ }^{(1,12)}$. Entre esses, verifica-se um personagem que foi responsável por formalizar a proposta de criação do Curso de Graduação. Este, docente aposentado pela Unicamp, deveria ter sido o primeiro sujeito a ser entrevistado, mas por dificuldades pessoais, uma vez que reside em outro município, foi o último a ser entrevistado.

A primeira entrevista foi realizada simultaneamente com duas professoras que apresentaram tal solicitação alegando que a interação, no momento da entrevista, favoreceria a rememoração dos fatos. Essa entrevista foi realizada na residência de uma das entrevistadas.

A entrevista seguinte foi realizada com uma das primeiras personagens desse momento histórico, atualmente integrante do corpo docente aposentado pela Unicamp, que a ocorrência de problemas de saúde impediu que the fosse concedida a prioridade temporal em relação às demais. Por escolha da depoente, a entrevista também se deu em sua residência.

O quarto depoente foi um aluno integrante da primeira turma de ingressantes no Curso de Enfermagem e que hoje faz parte do corpo docente do Departamento de Enfermagem desta Universidade. Essa escolha deveu-se ao fato de que no decorrer das entrevistas anteriores identificou-se a necessidade de inserir a perspectiva de um protagonista que colaborou na construção desse momento histórico na posição de aluno A entrevista ocorreu a partir de um segundo agendamento em virtude de impedimentos pessoais do depoente. A entrevista se deu nas próprias instalações do Departamento de Enfermagem, por escolha do entrevistado.

Ao longo desse período continuamos tentando estabelecer contatos com o professor que deveria ter sido o entrevistado inicial.

Ao longo dos relatos obtidos, todos os entrevistados mencionaram 0 nome de uma enfermeira que também participou do momento inicial de implantação do Curso, muito embora não tenha sido jamais contratada como docente. Assim, partimos à busca de alguma forma de contato com essa enfermeira citada. Com bastante dificuldade localizamos um número telefônico, mas após inúmeras tentativas não obtivemos contato. Dada à freqüente convergência na indicação do nome dessa profissional, entendemos a necessidade e importância de sua contribuição na história da instalação do Curso de Graduação em Enfermagem, assim, as novas tentativas de contato resultaram sucesso na obtenção do depoimento dessa profissional.

O sexto entrevistado foi um professor, também docente em exercício no Departamento de Enfermagem da FCM, Unicamp, que segundo informações obtidas nos documentos consultados, teria sido o sétimo integrante desse Departamento. Essa entrevista foi bastante breve, se comparadas às demais, muito embora esse docente tenha referido a posse de diversos documentos pertinentes a esse período histórico e também sua dedicação ao registro de sua memória pessoal relativa ao tempo de exercício docente nesta instituição.

A última entrevista foi realizada com o docente, aposentado pela Unicamp que foi o fundador do Curso de Graduação em Enfermagem. Houve várias tentativas anteriores a esta, mas sem sucesso, devido a disponibilidade pessoal do entrevistado. A entrevista ocorreu nas dependências do Hospital das Clínicas.

Diante dos objetivos propostos e dos acréscimos realizados nesse momento de coleta de dados, faltou entrevistar uma ex-docente, protagonista também fundamental para a reconstrução da história do Curso de Graduação em Enfermagem da Unicamp. Tratava-se de uma enfermeira já contratada, na época, como docente da FCM e responsável por ministrar disciplina de procedimentos de enfermagem para o curso médico, não entrevistada em decorrência do insucesso nas inúmeras tentativas de estabelecimento de contatos e posterior constatação de que a mesma transferira temporariamente sua residência para outro município e por motivo de doença que também a impedia de disponibilizar-se para entrevista. O limite cronológico para a finalização deste estudo definiu a desistência desse depoimento.

Todas as entrevistas realizadas foram transcritas em forma datilográfica e, em seguida, submetidas à conferência de fidelidade, ou seja, os textos transcritos foram comparados às respectivas gravações para as devidas correções. O passo seguinte consistiu na limpeza do texto para abolir as repetições, corrigir eventuais erros de português e inserir pontuações, sem afetar a forma e o sentido do texto.

Os textos originados das transcrições das fitas foram cuidadosamente lidos e re-lidos, visando obter um nível de atenção flutuante que permitiu a segura decomposição do material, a extração de recortes com a finalidade de utilizar, do conteúdo, apenas aquilo que era compatível com a síntese que se buscava, tomando por referência os objetivos do estudo.

Os conteúdos dos textos foram cotejados com dados originários de outras fontes de pesquisa, neste caso, os documentos institucionais já disponíveis, visto que outros documentos não se tornaram acessíveis no decorrer do processo de pesquisa, muito embora alguns entrevistados tenham feito menção à existência de outros documentos.

\section{RECONHECIMENTO DA HISTÓRIA CONSTRUÍDA}

Na década de 70, o Dr. Luiz Cietto, doutor e livre-docente pela Escola de Enfermagem Ana Neri da Universidade Federal do Rio de Janeiro, já desenvolvia atividades docentes na Universidade de Mogi das Cruzes, como professor titular. Tendo tido interesse em ingressar em uma universidade pública, com vistas a ampliar suas possibilidades de desenvolvimento acadêmico e científico na área de administração de enfermagem, submeteu-se e foi aprovado em concursos públicos para professor titular em uma universidade pública do estado do Paraná. 
Paralelamente, o Dr. Cietto em contato com Dr. Manoel Gonçalves Ferreira Filho, professor titular da Faculdade de Direito da USP e vicegovernador do Estado de São Paulo naquele momento, com vistas a identificar possibilidades de atuação docente no cenário de ensino universitário público paulista, recebeu a recomendação de procurar a Unicamp. Assim, o Dr. Cietto estabeleceu interação com o professor José Aristodemo Pinotti que, ocupando a direção da FCM, demonstrou grande interesse em sua contratação tendo em vista o plano de implantação do curso de enfermagem, formalmente previsto desde 1966, e a constatação de que 0 interessado apresentava titulação acadêmica e experiência docente compatíveis com o perfil desejado pela universidade. O professor Pinotti, empenhou-se na contratação do Dr. Cietto junto ao reitor da Unicamp. O sucesso dessa iniciativa levou o Dr. Cietto a desistir da vaga na universidade paranaense.

Em 03 de agosto de 1976, o Dr. Luiz Cietto foi, então, nomeado pelo reitor da Unicamp, Prof. Dr. Zeferino Vaz, como Professor Colaborador junto à FCM e também designado pelo diretor da FCM, Prof. Dr. José Aristodemo Pinotti, como presidente da Comissão de Implantação do Curso Superior de Enfermagem da universidade. Esta comissão era composta por outros nove integrantes, entre eles, sete docentes, a Chefe do Serviço de Enfermagem do Hospital das Clínicas e uma funcionária administrativa $^{(1)}$.

Aos 17 de novembro do mesmo ano, o Prof. Dr. Luiz Cietto encaminha ao Diretor da FCM da Unicamp, o Projeto de Implantação do Curso Superior de Enfermagem. No ofício de apresentação de tal projeto, o Prof. Luiz Cietto refere que o mesmo foi elaborado em curto período de tempo, três meses, em atendimento às determinações da própria direção da FCM.

Tal projeto compunha-se apenas do bloco de disciplinas do chamado tronco básico do curso, em virtude da necessidade de empreender-se urgente tramitação do mesmo na universidade ${ }^{(1)}$ e teria sido formulado, essencialmente, pelo empenho do Dr. Cietto que visitou algumas instituições, tais como a Escola Ana Nery e a Escola de Enfermagem da USP-SP, para apreender a orientação filosófica, a grade curricular e outros aspectos relevantes para a elaboração do projeto da enfermagem na Unicamp. A escolha dessas instituições, pelo depoimento do Professor Cietto, decorreu de suas relações anteriores com as mesmas.

Pelos depoimentos colhidos, identificou-se que nenhum dos sujeitos entrevistados participou da elaboração do projeto de criação do curso e que houve a colaboração da então assessora pedagógica da FCM, Sra. Sílvia Véspoli, na formulação das ementas das disciplinas que comporiam 0 curso ${ }^{(13)}$.

Em data não precisa, durante o ano de 1977, o Professor Dr. Luiz Cietto passa a assumir também a direção da Divisão de Enfermagem do Hospital das Clínicas (HC) da Unicamp, que até então se constituía como Serviço de Enfermagem, e permaneceu nessa função até o ano de 1980. Assim, esse professor acumulou, por um certo período, a direção da Divisão de Enfermagem do $\mathrm{HC}$, a responsabilidade pela implantação do Curso de Graduação, bem como sua coordenação.

É importante ressaltar que até o ano de 1985 o HC, Unicamp, mantevese nas instalações na Santa Casa de Misericórdia de Campinas. À frente da Divisão de Enfermagem do HC, o Prof. Dr. Luiz Cietto pôde aproximarse dos enfermeiros pertencentes ao quadro de funcionários da Unicamp e, assim, identificar, entre esses, aqueles que julgava apresentar o perfil por ele desejado. O interesse pelo ensino, a disponibilidade em inserir-se em carreira docente foram as características que Ihe pareciam adequadas aos candidatos a assumirem a responsabilidade de enfrentar os desafios da implantação do Curso de Enfermagem diante das circunstâncias postas.

Portanto, os enfermeiros, futuros docentes, não foram submetidos à qualquer tipo de seleção formal. Alguns foram convidados pelo Prof. Cietto, outros, espontaneamente, manifestaram interesse. No entanto, alguns entrevistados referiram que o convite que lhes fora feito apresentou-se com característica de determinação superior, sem possibilidade de recusa.

Sabe-se que, naquele momento, já havia uma docente, enfermeira e mestre, Profa Leonízia Rosa Moura de Toledo Tobar, nos quadros da FCM, que tinha a incumbência de ministrar disciplina de procedimentos e técnicas de enfermagem para o curso médico. Esta docente teve participação no planejamento de disciplinas, a partir da grade curricular elaborada pelo Prof. Dr. Luiz Cietto, e colaborou no ensino de disciplina de enfermagem no primeiro ano da graduação. Entretanto, provavelmente no ano de 1979 ou 1980, essa docente transferiu-se para outra unidade de ensino da universidade.

A enfermeira Maria José dos Anjos, mestranda na ocasião e professora do Curso de Auxiliar de Enfermagem do HC, também compartilhou da história prévia à criação do curso, auxiliando a professora Leonízia na disciplina de enfermagem oferecida ao curso de Medicina e também ministrando aulas de Licenciatura para enfermeiros do $\mathrm{HC}$ interessados em seguir carreira acadêmica. O Curso de Auxiliar foi criado em 01 de agosto de 1973 e funcionava mediante convênio da Unicamp com o Centro Médico de Campinas e a empresa Robert Bosch do Brasil. Após curto período de tempo, diante de algumas divergências de pensamento e postura com o coordenador do Curso de Graduação, não superadas, ela se afastou da graduação e reassumiu suas atividades didáticas no Curso de Auxiliar de Enfermagem.

Outra participante, também enfermeira do $\mathrm{HC}$, que auxiliou no processo de implantação do curso foi Dalva Maria Darcoletto Silva Pereira, que, sob a direção do Prof. Cietto passou a assumir a função de assistente administrativa da Divisão de Enfermagem do hospital. Nessa época, juntamente com a professora Leonízia, desenvolveu o planejamento das disciplinas do curso.

A aprovação do funcionamento do Curso Superior de Enfermagem pelo Conselho Diretor da Unicamp, bem como a devida autorização para a realização do concurso vestibular que ficaria naquele ano sob responsabilidade da FCM, sob supervisão da Câmara Curricular e Diretoria Acadêmica, ocorreu em 10 de novembro de $1977^{(1)}$.

Em 1978, acontece o primeiro vestibular e inicia-se o curso de Graduação em Enfermagem da FCM, nas modalidades bacharelado e licenciatura, no dia 01 de março, oferecendo 30 vagas e com um corpo docente composto apenas por três professores: Dr. Luiz Cietto, Leonízia Toledo Tobar e Dalva Maria Darcoletto Silva Pereira. Assim, as duas disciplinas de enfermagem iniciais foram ministradas por esses professores e a disciplina de nutrição foi ministrada pela nutricionista Myrta T. de Lima e Silva, da Prefeitura Municipal de Campinas, na condição de colaboradora voluntária. Esta profissional foi inserida informalmente para substituir outra nutricionista da universidade que assumiria o ensino dessas disciplinas, mas que, por razões de ordem pessoal, precisou afastar-se de suas atividades.

De acordo com o currículo em vigor na ocasião, grande parte das disciplinas, do primeiro ano, eram ministradas pelo Instituto de Biologia. À medida que as disciplinas específicas de enfermagem iam surgindo, houve a necessidade de inserção ou de contratação de outros professores.

No final do ano de 1978, devido às necessidades decorrentes das disciplinas que iam sendo paulatinamente oferecidas, outras enfermeiras do $\mathrm{HC}$ foram compondo o reduzido grupo de professores, entre elas, Rachel Noronha e Neusa Maria Costa Alexandre. Essas professoras, juntamente com Dalva, foram responsáveis pelas disciplinas de Médico Cirúrgica I e II, ministradas no segundo ano. No segundo semestre desse mesmo ano, houve também a participação da enfermeira Maria Euridéia de Castro para ministrar o ensino da disciplina de Enfermagem em Centro Cirúrgico, esta também permaneceu no curso por pouco tempo.

No ano de 1980, a enfermeira Maria Cecília Cardoso Benatti, também enfermeira do $\mathrm{HC}$, tendo recém concluído curso de especialização em Administração Hospitalar na Universidade de São Paulo, SP, passou a integrar o grupo de professores e assumiu as disciplinas de Enfermagem Ginecológica e Obstétrica e, no ano seguinte as disciplinas de Enfermagem 
em Saúde Pública e de Administração Aplicada à Enfermagem. Segundo relatos, devido à escassez de professores no curso, a enfermeira Maria Cecília Cardoso Benatti participou no ensino de 14 diferentes disciplinas. Também no ano de 1980, a enfermeira Keila Esmeralda Montebello Sabóya Brito, teve participação em disciplinas do campo da saúde da mulher, mas não permaneceu no quadro docente. Nesse mesmo ano, o enfermeiro José Franscisco Filho, também originário do $\mathrm{HC}$, assumiu o ensino de disciplinas de Enfermagem em Moléstias Infecciosas e, no ano seguinte, as de Enfermagem Psiquiátrica.

No ano de 1980 houve as primeiras contratações de enfermeiras na carreira docente, as professoras Anna de Lucca Oliveira e Eliana Russomano Veiga Sgambatti, a primeira para a área de enfermagem materno-infantil e a segunda para as disciplinas de Enfermagem Psiquiátrica. Segundo depoimentos colhidos, os outros enfermeiros, Dalva, José Francisco, Neusa, Maria Cecília e Rachel Noronha migraram para a carreira docente em 13 de novembro de 1980 e a criação do Departamento de Enfermagem foi aprovado pelo Conselho Diretor da Unicamp, em 10 de março de 1981. Em 30 de abril do mesmo ano, por Ato de Reitor, os docentes, relacionados a seguir, foram lotados no Departamento recém criado, Anna de Lucca Oliveira, Dalva Maria Darcoletto Silva Pereira, Eliana Russomano Veiga Sgambatti, José Francisco Filho, Keila Esmeralda Montebello Sabóya Brito, Leonízia Rosa Moura de Toledo, Luiz Cietto, Maria Cecília Cardoso Benatti, Neusa Maria Costa Alexandre e Rachel Noronha.

\section{REFERÊNCIAS}

1. Universidade Estadual de Campinas. Implantação do Curso de Graduação em Enfermagem da Faculdade de Ciências Médicas. Processo Unicamp n 312/77. Campinas (SP): Editora da Unicamp; 1977.

2. Universidade Estadual de Campinas. Unicamp 35 anos: ciência e tecnologia na imprensa. Campinas (SP): Editora da Unicamp; 2001.

3. Associação de Docentes da Unicamp. Adunicamp: 25 anos Autonomia - democracia - participação. Campinas (SP): Adunicamp; 2002.

4. Brasil. Ministério da Saúde. Fundação Serviços de Saúde Pública. Enfermagem - legislação e assuntos correlatos. $3^{\mathrm{a}}$ ed. Rio de Janeiro (RJ): Artes Gráficas da FSESP; 1974.

5. Universidade Estadual de Campinas. Catálogo dos cursos de graduação. Campinas (SP): Unicamp; 1996.

6. Pereira DMDS, Cerqueira LT. O Departamento de Enfermagem da FCM - Unicamp. Revista 30 anos FCM 1983: (esp): 27-8.

7. Nozawa MR. Perfil profissional, discurso e práticas de enfermeiras graduadas na Unicamp (tese). São Paulo (SP): Faculdade de Saúde Pública, Universidade de São Paulo; 1997.

8. 8.Nozawa MR, Kirschbaum DIR, Silva MAPD, Silva EM. Ensino de graduação em enfermagem da Unicamp: políticas e práticas. Rev Bras Enferm 2003; 56(6): 683-6.

9. Silva MAPD, Silva EM, Nozawa MR. Educação Profissional em Enfermagem e as relações com a graduação. Rev Paul Enferm 2001; 20(3): 5-10.

10. Freitas SM. História oral: possibilidades e procedimentos. São Paulo (SP): Imprensa Oficial do Estado; 2002.

11. Queiroz MIP. Relatos orais: do "indizível" ao "dizível". Ciência e Cultura 1987; 39(3): 272-86.

12. Universidade Estadual de Campinas. Implantação do Departamento de Enfermagem da Faculdade de Ciências Médicas. Processo Unicamp n 1099/80. Campinas (SP): Editora Unicamp; 1980.

13. Costallat LTL, organizador. Livro de memórias da FCM - Unicamp. Campinas (SP): Editora Unicamp; 2004. 